\title{
Comparative Evaluation of Rice SSR Markers on Different Oryza Species
}

\author{
Getachew Melaku1*, Shilai Zhang ${ }^{1}$ and Teklehaymanot Haileselassie ${ }^{2}$ \\ ${ }^{1}$ Research Center of Perennial Rice Engineering and Technology in Yunnan, School of Agriculture, Yunnan University, \\ Kunming 650091, China \\ ${ }^{2}$ Institute of Biotechnology, College of Natural and Computational Sciences, Addis Ababa University, Ethiopia
}

\begin{abstract}
The growing number of rice microsatellite markers permit a comprehensive comparison of allelic variation among the markers developed using different methods, with diverse repeat motifs and at variable genomic regions. Under this study, comparison between a set of 67 microsatellite markers representing the whole (twelve) rice chromosomes was done over worldwide collections of nine species of the genus Oryza. These SSR markers were evaluated for the genetic parameters such as; number of alleles amplified per primers, observed heterozygosity, gene diversity, rare allelic frequency and Polymorphic Information Content (PIC) values. Among the microsatellite markers that were assessed in the present study, highest overall degree of genetic diversity was recorded on a dinucleotide repeat motif containing markers. Therefore, employing such very informative markers for future molecular characterization and diversity study of Oryza species is advisable. The unique alleles generated from those polymorphic markers could also have significant role on the efforts of conservation, population genetics study and identification of both wild and cultivated Oryza species.
\end{abstract}

\section{Keywords}

Genetic diversity, Microsatellite markers, Oryza, Simple sequence repeats (SSR)

Abbreviations

F: Fixation Index; $F_{\text {is }}:$ Wrights Fixation Index; $F_{\text {it }}$ Total Inbreeding Coefficient; $F_{\text {st }}$ : Genetic Differentiation; He: Expected Heterozygosity; Ho: Observed Heterozygosity; Na: Actual Number of Alleles; Ne: Effective Number of Alleles; PIC: Polymorphic Information Content; PCR: Polymerase Chain Reaction; RM: Rice Marker; SSR: Simple Sequence Repeats

\section{Introduction}

The Asian cultivated rice (Oryza sativa L.) which feeds more than one third of the world's population is categorized under the genus Oryza [1]. A variety of local landraces and cultivars of rice are found in the indica and japonica subspecies [2,3]. Other than these two huge reservoirs of rice germplasm, over 20 wild Oryza species constitute an exceptionally valuable gene pool for rice improvement [4]. Thus, knowledge on the level of genetic diversities and species relationships in the genus Oryza is essential for efficient strategies targeting collection, conservation and introgression of useful genes to cultivated rice [5]. In rice breeding, hybridization between parental lines with a defined genetic distance and subsequent selection is a common approach [1]. These days, information on the extent of such genetic relationships between genotypes for an effective breeding program is generated from diverse molecular marker-based techniques [6].

Molecular markers are powerful tools in the assessment of genetic variation and elucidation of genetic relationships within and among plant species [7-9]. Likewise, molecular markers have been used in the genus Oryza to identify accessions [10], determine genetic structure and pattern of diversity [11], and optimize assembly of core collections [12]. Earlier molecular marker types such as RAPD, ISSR and AFLP have been used very frequently for fingerprinting and characterization of varieties and germplasm accessions [13]. However, they can be utilized without prior genomic information on the target crop and thus considered as markers of choice [6]. Since 2000, the locus specific Simple Sequence Repeat (SSR) markers got preferential application in cultivar identification of many crops such as, rice [14].

*Corresponding author: Getachew Melaku, Research Center of Perennial Rice Engineering and Technology in Yunnan, School of Agriculture, Yunnan University, Kunming 650091, China

Accepted: November 19, 2018

Published online: November 21, 2018

Citation: Melaku G, Zhang S, Haileselassie T (2018) Comparative Evaluation of Rice SSR Markers on Different Oryza Species. J Rice Res Dev 1(1):38-48 
SSRs are highly frequent 1 to 6 bp repeated motifs distributed throughout the nuclear [15], chloroplast $[16,17]$ and mitochondrial [18] genomes. Unique simple sequence repeat (SSR) profiles in rice cultivars can be generated by using few primers covering all of the 12 chromosomes [19]. However, a total of 18,828 Class $1 \mathrm{di}-$, tri- and tetranucleotide SSRs, representing 47 distinctive motif families, were identified and annotated on the rice genome [1]. The published high-density linkage map indicated abundance of microsatellite markers (with an average of 51 hypervariable SSRs per $\mathrm{Mb}$ ) with the highest density of markers at chromosome 3 (55.8 SSRMb-1) and at chromosome 4 (41.0 SSRMb-1) [20].

Such neutral and co-dominant SSR markers have merits like fast assay, technical simplicity, high polymorphism and stability [21]. Besides, the reproducible and cross-species transferable feature of SSR markers make them as valuable tools for genetic diversity study [9]. Among the most widely used DNA marker types, SSR markers are widely applied in gene mapping $[22,23]$, establishment of genetic relationships $[15,24]$, construction of fingerprints $[23,25]$, genetic purity test [26], molecular evolution studies [21], varietal identification and heterosis utilization [9]. For instance, clustering data generated from SSR makers were used for identifying a hybrid with highest heterosis from the intercrossing between different and distantly related Oryza species [1].

Variations on the molecular basis of polymorphism and distribution across the genome allow the different SSR marker types with different views for a given population structure [6]. The high degree of length polymorphism or variable allelic size by different SSR markers may also be attributed to chromosomal rearrangements during the genome's evolution, the different number of repeats in the SSR regions or replication slippage mechanism [21]. Differences in the level of SSR polymorphism may also be associated with gene conservation, source of the SSRs (genomic and EST-SSR) and/ or nature of the SSRs' nucleotide repeat unit (such as di-, triand tetra) [6]. Besides, [27] reported markers having perfect dinucleotide (GA and $\mathrm{CT}$ ) repeat motifs as markers with high level of variation among rice genotypes.

Many studies over diverse types of molecular markers reported SSRs as markers with highly significant allelic variation [28]. Allelic variation for tested SSR loci defines polymorphism among varieties or the polymorphism information content (PIC) value [1]. Hence, utility of SSR markers for assessment of genetic diversity, population structure study and crop improvement depend on quality of PIC they provide [6]. Indirectly, prior choice of markers with highly informative and polymorphic rice SSR markers-based study is too critical to conserve rice genotypes, reveal gene pool of rice landraces and unlock valuable genes for breeding purposes [29]. Thus, the present study was conducted to compare the widely used 67 selected rice SSR markers (RMs) for the assessment of genetic variability and population structure among 426 samples of 9 Oryza species. SSR markers evaluation over worldwide Oryza species collections for statistical and genetic parameters such as number of alleles amplified per primers, gene diversity, heterozygosity and PIC value identified markers which are more informative and promising for future genetic investigation on both wild and cultivated rice types.

\section{Materials and Methods}

\section{Plant material}

A total of 426 rice samples, hereafter referred as "accessions" comprising 35 accessions of cultivated rice germplasms from Yunnan University gene bank (Table 1), 31 accessions of 7 AA and CC genome wild Oryza species from IRRI (Table 2) and 360 collections from 12 Ethiopian populations of the African wild rice (O. longistaminata) (Table 3) were included in this study.

Table 1: 35 varieties of cultivated rice.

\begin{tabular}{|l|l|l|l|l|}
\hline Sample ID & Species/Subspecies & Genome type & Accession code & Origin \\
\hline P01 & Japonica & AA & Nippobrane & Yunnan university gene bank \\
\hline P02 & Japonica & AA & zhonghua11 & Yunnan university gene bank \\
\hline P03 & Japonica & AA & Kitaake & Yunnan university gene bank \\
\hline P04 & Indica & AA & RD23 & Yunnan university gene bank \\
\hline P05 & Indica & AA & minghui63 & Yunnan university gene bank \\
\hline P06 & Japonica & AA & Koshihikari & Yunnan university gene bank \\
\hline P07 & Japonica & AA & yundao2 & Yunnan university gene bank \\
\hline P08 & Japonica & AA & chujing27 & Yunnan university gene bank \\
\hline P09 & Indica & AA & 9311 & Yunnan university gene bank \\
\hline P10 & Indica & AA & luyin46 & Yunnan university gene bank \\
\hline P11 & Indica & AA & Huanghuazhan & Yunnan university gene bank \\
\hline P12 & Japonica & AA & IRAT104 & Yunnan university gene bank \\
\hline P13 & Indica & AA & IR64 & Yunnan university gene bank \\
\hline P14 & Indica & AA & guichao2 & Yunnan university gene bank \\
\hline P15 & Japonica & AA & Xueheaizao & Yunnan university gene bank \\
\hline P16 & Japonica & AA & Hejiang19 & Yunnan university gene bank \\
\hline P17 & Japonica & AA & Pokkali & Yunnan university gene bank \\
\hline P18 & Indica & AA & IR29 & Yunnan university gene bank \\
\hline P19 & Indica & AA & Mengwanggu & Yunnan university gene bank \\
\hline
\end{tabular}


Citation: Melaku G, Zhang S, Haileselassie T (2018) Comparative Evaluation of Rice SSR Markers on Different Oryza Species. J Rice Res Dev $1(1): 38-48$

\begin{tabular}{|l|l|l|l|l|}
\hline P20 & Indica & AA & taiwanludao1 & Yunnan university gene bank \\
\hline P21 & Japonica & AA & chujing28 & Yunnan university gene bank \\
\hline P22 & Japonica & AA & dianjingyou5 & Yunnan university gene bank \\
\hline P23 & Indica & AA & wendao12 & Yunnan university gene bank \\
\hline P24 & Japonica & AA & hexi41 & Yunnan university gene bank \\
\hline P25 & Indica & AA & yunhui290 & Yunnan university gene bank \\
\hline P26 & Indica & AA & banna21 & Yunnan university gene bank \\
\hline P27 & Japonica & AA & Akitakomati & Yunnan university gene bank \\
\hline P28 & Japonica & AA & Akihikari & Yunnan university gene bank \\
\hline P29 & Indica & AA & dianrui449 & Yunnan university gene bank \\
\hline P30 & Indica & AA & diantun502 & Yunnan university gene bank \\
\hline P31 & Japonica & AA & PR23 & Yunnan university gene bank \\
\hline P37 & Japonica & AA & yunjing37 & Yunnan university gene bank \\
\hline P41 & Indica & AA & mengwangmaxiangu & Yunnan university gene bank \\
\hline P42 & Indica & AA & Mengwangdabaigu & Yunnan university gene bank \\
\hline P43 & Indica & AA & mengwangxiaobaigu & Yunnan university gene bank \\
\hline
\end{tabular}

Table 2: 35 accessions representing 10 wild Oryza species and 1 weedy rice.

\begin{tabular}{|c|c|c|c|c|}
\hline Sample ID & Species & Genome type & Accession No & Origin \\
\hline YSD16 & O. barthii & $A A$ & Acc.100921 & \\
\hline WR23 & O. barthii & AA & Acc.104061 & Niger \\
\hline WR26 & O. barthii & AA & Acc.104284 & Mali \\
\hline YSD88 & O. glumaepatula & $A A$ & Acc.100894(W) & Cuba \\
\hline YSD90 & O. glumaepatula & AA & Acc.103812(W) & Brazil \\
\hline YSD92 & O. glumaepatula & $A A$ & Acc.105661 & Brazil \\
\hline YSD95 & O. glumaepatula & $A A$ & Acc.105662 & Brazil \\
\hline WR88 & O. glumaepatula & AA & Acc.100894(D) & Cuba \\
\hline WR90 & O. glumaepatula & AA & Acc.103812(D) & Brazil \\
\hline YSD64 & O. nivara & $A A$ & Acc. 80611 & India \\
\hline YSD66 & O. nivara & $A A$ & Acc.80677 & India \\
\hline WR60 & O. nivara & AA & Acc. 80432 & India \\
\hline WR65 & O. nivara & AA & Acc. 80625 & India \\
\hline WR77 & O. nivara & AA & Acc.80696 & India \\
\hline WR78 & O. nivara & AA & Acc. 81855 & India \\
\hline YSD42 & O. rufipogon & $A A$ & Acc. 82040 & Thailand \\
\hline YSD46 & O. rufipogon & $A A$ & Acc.106133(W) & India \\
\hline YSD52 & O. rufipogon & $A A$ & PCR9607(D) & Thailand \\
\hline WR40 & O. rufipogon & AA & Acc.81994 & Papua New Guinea \\
\hline WR46 & O. rufipogon & $A A$ & Acc.106133 & India \\
\hline WR48 & O. rufipogon & AA & Acc. 106340 & Myanmar \\
\hline WR55 & O. rufipogon open pollinated & AA & Acc.105832(W) & Bgo2,1998 gkanlaus \\
\hline WR57 & O. rufipogon open pollinated & $A A$ & Acc.106138(W) & Bgo2,2001 gkanlaus \\
\hline YSD55 & O. rufipogon open pollinated & AA & Acc.105832(D) & Bgo2,1998 gkanlaus \\
\hline YSD57 & O. rufipogon open pollinated & AA & Acc.106138(D) & Bgo2,2001 gkanlaus \\
\hline Lg & O. longistaminata & AA & & Niger \\
\hline YSD01 & O. officinalis & CC & 8712 & Sukhothai \\
\hline YSD05 & O. rhizomatis & CC & W95018(W) & IRRI \\
\hline WR05 & O. rhizomatis & $\mathrm{CC}$ & W95018(D) & IRRI \\
\hline WR03 & O. eichingeri & CC & Acc.105181(W) & Uganda \\
\hline YSD03 & O. eichingeri & $\mathrm{CC}$ & Acc.105181(D) & Uganda \\
\hline YSD08 & O. latifolia & CCDD & Acc.100169 & Costa Rice \\
\hline WR09 & O. australiensis & $\mathrm{EE}$ & Acc.101410(W) & Australia \\
\hline YSD09 & O. australiensis & $\mathrm{EE}$ & Acc.101410(D) & Australia \\
\hline YSD58 & Japonica weedy type & & 96036 & Korea \\
\hline
\end{tabular}




\section{Genomic DNA extraction and polymerase chain reaction (PCR)}

Total genomic DNA was extracted from fresh leaves by using CTAB protocol as described by [30]. Quality for the extracted DNA was determined by electrophoresis in a $1 \%$ agarose gel and quantification was accomplished using a spectrophotometer. For SSR analyses, the extracted DNA samples were diluted to $20 \mathrm{ng} / \mu \mathrm{l}$ using TE (Tris-EDTA) and stored at $-20^{\circ} \mathrm{C}$.

A total of Sixty-seven nuclear SSR markers covering the 12 rice chromosomes were used in this study (Table 4). Those polymorphic SSR markers were selected for amplification according to the reports from [27,31,32]. Polymerase chain reaction (PCR) was done by using a $10 \mu \mathrm{l}$ reaction mixture in a 96-well plate. Each reaction mixture contained $4 \mu \mathrm{l}$

Table 3: Ecology of the Ethiopian O. longistaminata populations.

\begin{tabular}{|c|c|c|c|c|c|}
\hline Group code & Site name & Coordinate 1 & Coordinate 2 & Elevation & Ecology type \\
\hline Gambella1 & $\begin{array}{l}\text { Gambella Agricultural } \\
\text { Research Institute }\end{array}$ & 36Р0673586 & UTM0912603 & $431 \mathrm{M}$ & $\begin{array}{l}\text { In a small stream with no rice cultivation } \\
\text { history }\end{array}$ \\
\hline Gambella2 & Kera & 36P0673826 & UTM0912320 & $438 \mathrm{M}$ & $\begin{array}{l}\text { A wetland which gets dry in a dry season and } \\
\text { without a rice cultivation practice }\end{array}$ \\
\hline Gambella3 & Elai & 36P0674462 & UTM0911700 & $442 M$ & $\begin{array}{l}\text { Baro river provides water and had rice } \\
\text { cultivation trial in } 2011\end{array}$ \\
\hline Gambella4 & Echewi & 36P0676012 & UTM0909521 & $443 \mathrm{M}$ & $\begin{array}{l}\text { A swampy area without any rice production } \\
\text { practice }\end{array}$ \\
\hline Gambella5 & Aleworo & 36N0667699 & UTM0872871 & $460 M$ & $\begin{array}{l}\text { An isolated area and free from any rice } \\
\text { cultivation }\end{array}$ \\
\hline Gambella6 & Catholic Church & $36 N 0667515$ & UTM0872896 & $457 \mathrm{M}$ & $\begin{array}{l}\text { A semi-wetland area without rice production } \\
\text { history }\end{array}$ \\
\hline Amhara1 & Tana Hotel & 37P0325270 & UTM1283260 & $1,800 \mathrm{M}$ & $\begin{array}{l}\text { Tana lake is the source of water and it is } \\
\text { an isolated area with no rice production } \\
\text { practice }\end{array}$ \\
\hline Amhara2 & Tewa Zakena & 37P0359496 & UTM1320023 & $1,801 \mathrm{M}$ & $\begin{array}{l}\text { A stream water near the road is the source } \\
\text { and there is a rice farm at } 100 \text { Meter away } \\
\text { from the collection site }\end{array}$ \\
\hline Amhara3 & Abwa Kokite School & 37P0359239 & UTM1324519 & $1,801 \mathrm{M}$ & $\begin{array}{l}\text { Many rice varieties by the Woreta } \\
\text { Agricultural Institute has been grown at } 50 \\
\text { M distance }\end{array}$ \\
\hline Amhara4 & Gami Gote & 37P0316212 & UTM1294335 & $1,790 \mathrm{M}$ & $\begin{array}{l}\text { Had a trial to cultivate rice in } 2011 \text { and } 2012 \\
\text { but the overflow of water from Tana lake } \\
\text { makes it less likely }\end{array}$ \\
\hline Amhara5 & Wenjeta Kebelle & 37P0314305 & UTM1291528 & $1,792 \mathrm{M}$ & $\begin{array}{l}\text { A rice farm from 2009-2013. Though, it is } \\
\text { now abandoned }\end{array}$ \\
\hline Amhara6 & Fessesa & 37P0313974 & UTM1292507 & $1,798 \mathrm{M}$ & A rice farm up to 2014 \\
\hline
\end{tabular}

Table 4: List of primers used in the PCR with their $5^{\prime}-3^{\prime}$ sequence, repeat motif's, chromosomal location and annealing temperature.

\begin{tabular}{|c|c|c|c|c|}
\hline Primer & 5'-3' sequence of FWD/REV primer & SSR repeat motif & Chromosomal location & Annealing temperature \\
\hline \multirow[t]{2}{*}{ RM5 } & FWD: TGCAACTTCTAGCTGCTCGA & \multirow[t]{2}{*}{ (GA)14 } & \multirow[t]{2}{*}{1} & \\
\hline & REV: GCATCCGATCTTGATGGG & & & 55 \\
\hline \multirow[t]{2}{*}{ RM6 } & FWD: TGCAACTTCTAGCTGCTCGA & \multirow[t]{2}{*}{ (AG)16 } & \multirow[t]{2}{*}{2} & \\
\hline & REV: TCGTCTACTGTTGGCTGCAC & & & 55 \\
\hline \multirow[t]{2}{*}{ RM11 } & FWD: TCTCCTCTTCCCCCGATC & \multirow[t]{2}{*}{ (GA)17 } & \multirow[t]{2}{*}{7} & \\
\hline & REV: ATAGCGGGCGAGGCTTAG & & & 55 \\
\hline \multirow[t]{2}{*}{ RM14 } & FWD: CCGAGGAGAGGAGTTCGAC & \multirow[t]{2}{*}{ (GA)17 } & \multirow[t]{2}{*}{1} & \\
\hline & REV: GTGCCAATTTCCTCGAAAAA & & & 55 \\
\hline \multirow[t]{2}{*}{ RM17 } & FWD: TGCCCTGTTATTTTCTTCTCTC & \multirow[t]{2}{*}{$(G A) 21$} & \multirow[t]{2}{*}{12} & \\
\hline & REV: GGTGATCCTTTCCCATTTCA & & & 55 \\
\hline \multirow[t]{2}{*}{ RM19 } & FWD: CAAAAACAGAGCAGATGAC & \multirow[t]{2}{*}{ (ATC)10 } & \multirow[t]{2}{*}{12} & \\
\hline & REV: CTCAAGATGGACGCCAAGA & & & 55 \\
\hline \multirow[t]{2}{*}{ RM21 } & FWD: ACAGTATTCCGTAGGCACGG & \multirow[t]{2}{*}{$(G A) 21$} & \multirow[t]{2}{*}{11} & \\
\hline & REV: GCTCCATGAGGGTGGTAGAG & & & 55 \\
\hline \multirow[t]{2}{*}{ RM22 } & FWD: GGTTTGGGAGCCCATAATCT & \multirow[t]{2}{*}{$(G A) 22$} & \multirow[t]{2}{*}{3} & \\
\hline & REV: CTGGGCTTCTTTCACTCGTC & & & 55 \\
\hline \multirow[t]{2}{*}{ RM23 } & FWD: CATTGGAGTGGAGGCTGG & \multirow[t]{2}{*}{ (GA)15 } & \multirow[t]{2}{*}{1} & \\
\hline & REV: GTCAGGCTTCTGCCATTCTC & & & 55 \\
\hline
\end{tabular}


Citation: Melaku G, Zhang S, Haileselassie T (2018) Comparative Evaluation of Rice SSR Markers on Different Oryza Species. J Rice Res Dev $1(1): 38-48$

\begin{tabular}{|c|c|c|c|c|}
\hline \multirow[t]{2}{*}{ RM24 } & FWD: GAAGTGTGATCACTGTAACC & \multirow[t]{2}{*}{$(\mathrm{GA}) 29$} & \multirow[t]{2}{*}{1} & \\
\hline & REV: TACAGTGGACGGCGAAGTCG & & & 55 \\
\hline \multirow[t]{2}{*}{ RM25 } & FWD: GGAAAGAATGATCCTTTTCATGG & \multirow[t]{2}{*}{ (GA)18 } & \multirow[t]{2}{*}{8} & \\
\hline & REV: CTACCATCAAAACCAATGTTC & & & 55 \\
\hline \multirow[t]{2}{*}{ RM26 } & FWD: GAGTCGACGAGCGGCAGA & \multirow[t]{2}{*}{ (GA)15 } & \multirow[t]{2}{*}{5} & \\
\hline & REV: CTGCGAGCGACGGTAACA & & & 55 \\
\hline \multirow[t]{2}{*}{ RM29 } & FWD: CAGGGACCCACCTGTCATAC & \multirow[t]{2}{*}{$(\mathrm{GA}) 7-18(\mathrm{GA}) 5(\mathrm{AG}) 4$} & \multirow[t]{2}{*}{2} & \\
\hline & REV: AACGTTGGTCATATCGGTGG & & & 55 \\
\hline \multirow[t]{2}{*}{ RM30 } & FWD: GGTTAGGCATCGTCACGG & \multirow[t]{2}{*}{ (AG)9A(AGA)12 } & \multirow[t]{2}{*}{6} & \\
\hline & REV: TCACCTCACCACACGACACG & & & 55 \\
\hline \multirow[t]{2}{*}{ RM31 } & FWD: GATCACGATCCACTGGAGCT & \multirow[t]{2}{*}{ (GA)15 } & \multirow[t]{2}{*}{5} & \\
\hline & REV: AAGTCCATTACTCTCCTCCC & & & 55 \\
\hline \multirow[t]{2}{*}{ RM42 } & FWD: ATCCTACCGCTGACCATGAG & $(\mathrm{AG}) 5(\mathrm{GA})(\mathrm{AG}) 2 \mathrm{~T}(\mathrm{GA}) \mathrm{A}(\mathrm{AG}) 5$ & 8 & \\
\hline & REV: TTTGGTCTACGTGGCGTACA & & & 55 \\
\hline RM44 & FWD: ACGGGCAATCCGAACAACC & $(\mathrm{GA}) 16$ & 8 & \\
\hline & REV: TCGGGAAAACCTACCCTACC & & & 55 \\
\hline RM49 & FWD: TTCGGAAGTTGGTTACTGATCA & $(\mathrm{GA}) 27$ & 3 & \\
\hline & REV: TTGGAGCGGATTCGGAGG & & & 55 \\
\hline RM50 & FWD: ACTGTACCGGTCGAAGACG & (CTAT)4(CT)15 & 6 & \\
\hline & REV: AAATTCCACGTCAGCCTCC & & & 55 \\
\hline RM55 & FWD: CCGTCGCCGTAGTAGAGAAG & $(\mathrm{GA}) 17$ & 3 & \\
\hline & REV: TCCCGGTTATTTTAAGGCG & & & 55 \\
\hline RM60 & FWD: AGTCCCATGTTCCACTTCCG & (AATT)5AATCT(AATT) & 3 & \\
\hline & REV: ATGGCTACTGCCTGTACTAC & & & 55 \\
\hline RM80 & FWD: TTGAAGGCGCTGAAGGAG & (TCT)25 & 8 & \\
\hline & REV: CATCAACCTCGTCTTCACCG & & & 55 \\
\hline RM84 & FWD: TAAGGGTCCATCCACAAGATG & (TCT)10 & 1 & \\
\hline & REV: TTGCAAATGCAGCTAGAGTAC & & & 55 \\
\hline RM105 & FWD: GTCGTCGACCCATCGGAGCCAC & (CCT)6 & 10 & \\
\hline & REV: TGGTCGAGGTGGGGATCGGGTC & & & 55 \\
\hline RM110 & FWD: TCGAAGCCATCCACCAACGAAG & $(\mathrm{GA}) 15$ & 2 & \\
\hline & REV: TCCGTACGCCGACGAGGTCGAG & & & 55 \\
\hline RM118 & FWD: CCAATCGGAGCCACCGGAGAGC & $(G A) 8$ & 7 & \\
\hline & REV: CACATCCTCCAGCGACGCCGAG & & & 67 \\
\hline RM125 & FWD: ATCAGCAGCCATGGCAGCGACC & $(\mathrm{GCT}) 8$ & 7 & 55 \\
\hline & REV: AGGGGATCATGTGCCGAAGGCC & & & \\
\hline RM126 & FWD: CGCGTCCGCGATAAACACAGGG & $(\mathrm{GA}) 7$ & 8 & \\
\hline & REV: TCGCACAGGTGAGGCCATGTCG & & & 55 \\
\hline RM129 & FWD: TCTCTCCGGAGCCAAGGCGAGG & & & \\
\hline & REV: CGAGCCACGACGCGATGTACCC & (CGG)8 & 1 & 55 \\
\hline RM133 & FWD: TTGGATTGTTTTGCTGGCTCGC & $(\mathrm{CT}) 8$ & 6 & \\
\hline & REV: GGAACACGGGGTCGGAAGCGAC & & & 60 \\
\hline RM134 & FWD: ACAAGGCCGCGAGAGGATTCCG & & & \\
\hline & REV: GCTCTCCGGTGGCTCCGATTGG & $(\mathrm{CCA}) 7$ & 7 & 55 \\
\hline RM136 & FWD: GAGAGCTCAGCTGCTGCCTCTAGC & $(A G G) 7$ & 6 & \\
\hline & REV: GAGGAGCGCCACGGTGTACGCC & & & 55 \\
\hline RM144 & FWD: TGCCCTGGCGCAAATTTGATCC & (ATT)11 & 11 & \\
\hline & REV: GCTAGAGGAGATCAGATGGTAGTGCATG & & & 57 \\
\hline RM152 & FWD: GAAACCACCACACCTCACCG & $(G G C) 10$ & 8 & \\
\hline & REV: CCGTAGACCTTCTTGAAGTAG & & & 55 \\
\hline RM159 & FWD: GGGGCACTGGCAAGGGTGAAGG & $(\mathrm{GA}) 19$ & 8 & \\
\hline & REV: GCTTGTGCTTCTCTCTCTCTCTCTCTCTC & & & 55 \\
\hline RM161 & FWD: TGCAGATGAGAAGCGGCGCCTC & (AG)20 & 5 & \\
\hline & REV: TGTGTCATCAGACGGCGCTCCG & & & 57 \\
\hline RM162 & FWD: GCCAGCAAAACCAGGGATCCGG & $(\mathrm{AC}) 20$ & 6 & \\
\hline & REV: CAAGGTCTTGTGCGGCTTGCGG & & & 61 \\
\hline
\end{tabular}


Citation: Melaku G, Zhang S, Haileselassie T (2018) Comparative Evaluation of Rice SSR Markers on Different Oryza Species. J Rice Res Dev $1(1): 38-48$

\begin{tabular}{|c|c|c|c|c|}
\hline \multirow[t]{2}{*}{ RM171 } & FWD: AACGCGAGGACACGTACTTAC & \multirow[t]{2}{*}{ (GATG)5 } & \multirow[t]{2}{*}{10} & \\
\hline & REV: ACGAGATACGTACGCCTTTG & & & 55 \\
\hline \multirow[t]{2}{*}{ RM184 } & FWD: ATCCCATTCGCCAAAACCGGCC & \multirow[t]{2}{*}{$(\mathrm{CA}) 7$} & \multirow[t]{2}{*}{10} & \multirow[t]{2}{*}{55} \\
\hline & REV: TGACACTTGGAGAGCGGTGTGG & & & \\
\hline \multirow[t]{2}{*}{ RM205 } & FWD: CTGGTTCTGTATGGGAGCAG & \multirow[t]{2}{*}{$(G A) 25$} & \multirow[t]{2}{*}{9} & \\
\hline & REV: CTGGCCCTTCACGTTTCAGTG & & & 55 \\
\hline \multirow[t]{2}{*}{ RM207 } & FWD: CCATTCGTGAGAAGATCTGA & \multirow[t]{2}{*}{ (CT)25 } & \multirow[t]{2}{*}{2} & \\
\hline & REV: CACCTCATCCTCGTAACGCC & & & 55 \\
\hline \multirow[t]{2}{*}{ RM209 } & FWD: ATATGAGTTGCTGTCGTGCG & \multirow[t]{2}{*}{ (CT)18 } & \multirow[t]{2}{*}{11} & \\
\hline & REV: CAАCTTGCATCCTCСССТCC & & & 55 \\
\hline \multirow[t]{2}{*}{ RM212 } & FWD: CCACTTTTCAGCTACTACCAG & \multirow[t]{2}{*}{$(G A) 24$} & \multirow[t]{2}{*}{1} & \\
\hline & REV: CACCCATTTGTCTCTCATTATG & & & 55 \\
\hline RM213 & FWD: ATCTGTTTGCAGGGGACAAG & (CT)17 & 2 & 55 \\
\hline & REV: AGGTCTAGACGATGTCGTGA & & & \\
\hline RM215 & FWD: CAAAATGGAGCAGCAAGAGC & (GA)16 & 9 & \\
\hline & REV: TGAGCACCTCCTTCTCTGTAG & & & 55 \\
\hline RM219 & FWD: CGTCGGATGATGTAAAGCCT & (GA)17 & 9 & \\
\hline & REV: CATATCGGCATTCGCCTG & & & 55 \\
\hline RM220 & FWD: GGAAGGTAACTGTTTCCAAC & (CT)17 & 1 & \\
\hline & REV: GAAATGCTTCCCACATGTCT & & & 55 \\
\hline RM224 & FWD: ATCGATCGATCTTCACGAGG & $(A A G) 8(A G) 13 G(A G)$ & 11 & \\
\hline & REV: TGCTATAAAAGGCATTCGGG & & & 55 \\
\hline RM225 & FWD: TGCCCATATGGTCTGGATG & (CT)18 & 6 & \\
\hline & REV: GAAAGTGGATCAGGAAGGC & & & 55 \\
\hline RM333 & FWD: GTACGACTACGAGTGTCACCAA & (TAT)19(СTT)19 & 10 & 55 \\
\hline & REV: GTCTTCGCGATCACTCGC & & & \\
\hline RM337 & FWD: GTAGGAAAGGAAGGGCAGAG & CTT64 8 (CTT)4-19-(CTT)8 & 8 & 55 \\
\hline & REV: CGATAGATAGCTAGATGTGGCC & & & \\
\hline RM153 & FWD: GCCTCGAGCATCATCATCAG & $(\mathrm{GAA}) 9$ & 5 & 55 \\
\hline & REV: ATCAACCTGCACTTGCCTGG & & & \\
\hline RM154 & FWD: ACCCTCTCCGCCTCGCCTCCTC & $(G A) 21$ & 2 & 61 \\
\hline & REV: CTCCTCCTCCTGCGACCGCTCC & & & \\
\hline RM164 & FWD: TCTTGCCCGTCACTGCAGATATCC & (GT)16TT(GT)4 & 6 & 55 \\
\hline & REV: GCAGCCCTAATGCTACAATTCTTC & & & \\
\hline RM180 & FWD: CTACATCGGCTTAGGTGTAGCAACACG & (ATT)10 & 7 & 55 \\
\hline & REV: ACTTGCTCTACTTGTGGTGAGGGACTG & & & \\
\hline RM206 & FWD: TAGTTTAACCAAGACTCTC & (GA)19 & 2 & 55 \\
\hline & REV: GGTTGAACCCAAATCTGCA & & & \\
\hline RM307 & FWD: GTACTACCGACCTACCGTTCAC & (AT)14(GT)21 & 4 & 55 \\
\hline & REV: CTGCTATGCATGAACTGCTC & & & \\
\hline RM322 & FWD: CAAGCGAAAATCCCAGCAG & (CAT)7 & 2 & 55 \\
\hline & REV: GATGAAACTGGCATTGCCTG & & & \\
\hline RM1 & FWD: GCGAAAACACAATGCAAAAA & (GA)26 & 1 & 55 \\
\hline & REV: GCGTTGGTTGGACCTGAC & & & \\
\hline RM211 & FWD: CCGATCTCATCAACCAACTG & $(\mathrm{TC}) 3 \mathrm{~A}(\mathrm{TC}) 18$ & 2 & 55 \\
\hline & REV: CTTCACGAGGATCTCAAAGG & & & \\
\hline RM251 & FWD: GAATGGCAATGGCGCTAG & (CT)29 & 3 & 55 \\
\hline & REV: ATGCGGTTCAAGATTCGATC & & & \\
\hline RM263 & FWD: CCCAGGCTAGCTCATGAACC & (CT)34 & 2 & 55 \\
\hline & REV: GCTACGTTTGAGCTACCACG & & & \\
\hline RM167 & FWD: GATCCAGCGTGAGGAACACGT & GGAA(GA)16GGGG & 11 & 55 \\
\hline & REV: AGTCCGACCACAAGGTGCGTTGTC & & & \\
\hline RM216 & FWD: TTCCCCAATGGAACAGTGAC & (CT)18 & 10 & 55 \\
\hline & REV: AGGGTCTACCACCCGATCTC & & & \\
\hline RM475 & FWD: CCTCACGATTTTCCTCCAAC & (TATC)8 & & \\
\hline & REV: ACGGTGGGATTAGACTGTGC & & 2 & 55 \\
\hline
\end{tabular}


Citation: Melaku G, Zhang S, Haileselassie T (2018) Comparative Evaluation of Rice SSR Markers on Different Oryza Species. J Rice Res Dev 1(1):38-48

\begin{tabular}{|l|l|l|l|l|}
\hline \multirow{2}{*}{ RM6344 } & FWD: ACACGCCATGGATGATGAC & (GAA)8 & 7 & 55 \\
\cline { 2 - 5 } & REV: TGGCATCATCACTTCCTCAC & & & \\
\hline RM335 & FWD: GTACACACCCACATCGAGAAG & (CTT)25 & 4 & 55 \\
\cline { 2 - 5 } & REV: GCTCTATGCGAGTATCCATGG & & & \\
\hline
\end{tabular}

Table 5: Genetic diversity indices revealed by 67 SSR markers on 430 wild, weedy and cultivated rice accessions.

\begin{tabular}{|c|c|c|c|c|c|c|c|c|c|c|c|}
\hline Marker type & Major Allele Frequency & No of rare alleles & Allele No & PIC & $\mathrm{Ne}$ & Ho & $\mathrm{He}$ & $F_{\text {is }}$ & $F_{i t}$ & $\mathbf{F}_{\text {st }}$ & $\mathrm{Nm}$ \\
\hline RM125 & 0.67 & 1 & 5 & 0.47 & 1.48 & 0.18 & 0.23 & 0.23 & 0.75 & 0.67 & 0.12 \\
\hline RM1 & 0.34 & 1 & 7 & 0.72 & 2.3 & 0.22 & 0.46 & 0.52 & 0.67 & 0.31 & 0.55 \\
\hline RM263 & 0.45 & 1 & 5 & 0.69 & 1.98 & 0.43 & 0.43 & 0.02 & 0.37 & 0.36 & 0.45 \\
\hline RM337 & 0.53 & 0 & 4 & 0.46 & 1.61 & 0.02 & 0.24 & 0.9 & 0.97 & 0.65 & 0.14 \\
\hline RM211 & 0.51 & 2 & 5 & 0.54 & 1.38 & 0.04 & 0.19 & 0.8 & 0.88 & 0.41 & 0.36 \\
\hline RM154 & 0.4 & 0 & 5 & 0.62 & 1.68 & 0.13 & 0.28 & 0.53 & 0.82 & 0.62 & 0.15 \\
\hline RM180 & 0.56 & 0 & 5 & 0.54 & 1.06 & 0.04 & 0.23 & 0.84 & 0.96 & 0.73 & 0.09 \\
\hline RM167 & 0.81 & 5 & 8 & 0.28 & 1.46 & 0 & 0.23 & 1 & 1 & 0.58 & 0.18 \\
\hline RM164 & 0.5 & 2 & 7 & 0.58 & 1.52 & 0.01 & 0.25 & 0.97 & 0.99 & 0.68 & 0.12 \\
\hline RM251 & 0.86 & 3 & 8 & 0.24 & 2 & 0.1 & 0.38 & 0.74 & 0.86 & 0.48 & 0.27 \\
\hline RM110 & 0.56 & 2 & 6 & 0.41 & 1.92 & 0.37 & 0.44 & 0.15 & 0.5 & 0.41 & 0.36 \\
\hline RM322 & 0.87 & 3 & 6 & 0.22 & 1.83 & 0.35 & 0.38 & 0.09 & 0.45 & 0.4 & 0.38 \\
\hline RM206 & 0.6 & 6 & 13 & 0.57 & 1.39 & 0.03 & 0.3 & 0.89 & 0.96 & 0.65 & 0.13 \\
\hline RM84 & 0.54 & 3 & 11 & 0.63 & 2.14 & 0.31 & 0.47 & 0.33 & 0.59 & 0.39 & 0.39 \\
\hline RM216 & 0.79 & 2 & 7 & 0.33 & 1.39 & 0.04 & 0.21 & 0.82 & 0.95 & 0.7 & 0.11 \\
\hline RM6344 & 0.52 & 5 & 9 & 0.55 & 1.67 & 0.12 & 0.3 & 0.59 & 0.85 & 0.62 & 0.15 \\
\hline RM307 & 0.86 & 1 & 7 & 0.24 & 1.7 & 0.08 & 0.27 & 0.69 & 0.89 & 0.64 & 0.14 \\
\hline RM333 & 0.52 & 1 & 9 & 0.49 & 1.96 & 0.09 & 0.33 & 0.74 & 0.89 & 0.58 & 0.18 \\
\hline RM153 & 0.9 & 1 & 6 & 0.18 & 1.55 & 0.07 & 0.25 & 0.71 & 0.9 & 0.65 & 0.13 \\
\hline RM5 & 0.74 & 3 & 9 & 0.42 & 1.66 & 0.19 & 0.33 & 0.43 & 0.76 & 0.58 & 0.18 \\
\hline RM475 & 0.36 & 2 & 5 & 0.65 & 0.88 & 0.05 & 0.2 & 0.77 & 0.95 & 0.78 & 0.07 \\
\hline RM6 & 0.37 & 1 & 8 & 0.61 & 1.64 & 0.05 & 0.31 & 0.83 & 0.93 & 0.58 & 0.18 \\
\hline RM11 & 0.76 & 4 & 6 & 0.38 & 1.65 & 0.04 & 0.3 & 0.87 & 0.93 & 0.49 & 0.26 \\
\hline RM17 & 0.54 & 0 & 4 & 0.53 & 1.49 & 0.2 & 0.29 & 0.31 & 0.68 & 0.54 & 0.21 \\
\hline RM19 & 0.92 & 1 & 4 & 0.14 & 1.13 & 0.01 & 0.12 & 0.89 & 0.98 & 0.83 & 0.05 \\
\hline RM21 & 0.47 & 5 & 7 & 0.64 & 1.47 & 0.07 & 0.26 & 0.73 & 0.88 & 0.54 & 0.21 \\
\hline RM23 & 0.44 & 4 & 8 & 0.60 & 1.45 & 0.03 & 0.24 & 0.87 & 0.96 & 0.67 & 0.12 \\
\hline RM24 & 0.67 & 1 & 4 & 0.40 & 1.65 & 0.03 & 0.31 & 0.91 & 0.95 & 0.51 & 0.24 \\
\hline RM25 & 0.41 & 1 & 6 & 0.60 & 1.51 & 0 & 0.29 & 1 & 1 & 0.67 & 0.13 \\
\hline RM42 & 0.46 & 3 & 5 & 0.53 & 1.26 & 0.01 & 0.18 & 0.92 & 0.97 & 0.57 & 0.19 \\
\hline RM31 & 0.5 & 3 & 7 & 0.62 & 1.62 & 0 & 0.28 & 1 & 1 & 0.57 & 0.19 \\
\hline RM30 & 0.74 & 2 & 7 & 0.41 & 1.58 & 0.01 & 0.27 & 0.97 & 0.99 & 0.62 & 0.15 \\
\hline RM29 & 0.91 & 2 & 5 & 0.15 & 1.34 & 0 & 0.19 & 1 & 1 & 0.73 & 0.09 \\
\hline RM26 & 0.86 & 1 & 4 & 0.23 & 1.37 & 0 & 0.21 & 1 & 1 & 0.67 & 0.12 \\
\hline RM22 & 0.94 & 0 & 2 & 0.10 & 0.89 & 0 & 0.13 & 1 & 1 & 0.83 & 0.05 \\
\hline RM14 & 0.61 & 1 & 3 & 0.37 & 0.85 & 0.12 & 0.17 & 0.31 & 0.86 & 0.8 & 0.06 \\
\hline RM171 & 0.91 & 0 & 2 & 0.15 & 1.09 & 0 & 0.13 & 1 & 1 & 0.79 & 0.07 \\
\hline RM335 & 0.53 & 4 & 7 & 0.42 & 1.21 & 0 & 0.11 & 0.98 & 1 & 0.82 & 0.06 \\
\hline RM44 & 0.44 & 2 & 8 & 0.64 & 1.86 & 0.17 & 0.38 & 0.55 & 0.78 & 0.52 & 0.23 \\
\hline RM49 & 0.86 & 3 & 5 & 0.23 & 0.9 & 0.11 & 0.15 & 0.28 & 0.84 & 0.78 & 0.07 \\
\hline RM50 & 0.39 & 2 & 5 & 0.60 & 1.3 & 0.03 & 0.2 & 0.84 & 0.95 & 0.71 & 0.1 \\
\hline RM55 & 0.8 & 1 & 4 & 0.31 & 1.42 & 0.11 & 0.23 & 0.53 & 0.63 & 0.21 & 0.95 \\
\hline RM60 & 0.95 & 0 & 3 & 0.09 & 1.35 & 0 & 0.14 & 1 & 1 & 0.7 & 0.11 \\
\hline RM80 & 0.79 & 1 & 9 & 0.36 & 2.19 & 0.3 & 0.45 & 0.32 & 0.61 & 0.42 & 0.34 \\
\hline RM105 & 0.37 & 0 & 5 & 0.63 & 1.67 & 0.19 & 0.35 & 0.44 & 0.71 & 0.48 & 0.27 \\
\hline RM118 & 0.66 & 4 & 7 & 0.52 & 1.27 & 0.07 & 0.18 & 0.63 & 0.89 & 0.7 & 0.11 \\
\hline RM126 & 0.73 & 2 & 4 & 0.38 & 1.1 & 0.02 & 0.06 & 0.68 & 0.96 & 0.88 & 0.03 \\
\hline RM129 & 0.58 & 3 & 6 & 0.41 & 1.42 & 0.07 & 0.23 & 0.7 & 0.9 & 0.67 & 0.12 \\
\hline RM133 & 0.48 & 5 & 7 & 0.43 & 1.18 & 0.09 & 0.09 & 0.07 & 0.67 & 0.64 & 0.14 \\
\hline RM144 & 0.81 & 1 & 5 & 0.30 & 1.37 & 0.03 & 0.25 & 0.86 & 0.95 & 0.66 & 0.13 \\
\hline
\end{tabular}


Citation: Melaku G, Zhang S, Haileselassie T (2018) Comparative Evaluation of Rice SSR Markers on Different Oryza Species. J Rice Res Dev $1(1): 38-48$

\begin{tabular}{|c|c|c|c|c|c|c|c|c|c|c|c|}
\hline RM152 & 0.5 & 3 & 8 & 0.60 & 1.71 & 0.09 & 0.32 & 0.71 & 0.87 & 0.55 & 0.2 \\
\hline RM134 & 0.82 & 0 & 4 & 0.26 & 1.41 & 0.15 & 0.24 & 0.38 & 0.64 & 0.42 & 0.35 \\
\hline RM136 & 0.81 & 2 & 7 & 0.29 & 1.77 & 0.5 & 0.31 & -0.6 & 0.27 & 0.55 & 0.21 \\
\hline RM159 & 0.89 & 3 & 7 & 0.19 & 1.77 & 0.41 & 0.37 & -0.1 & 0.47 & 0.52 & 0.23 \\
\hline RM161 & 0.92 & 0 & 4 & 0.14 & 1.44 & 0.08 & 0.26 & 0.68 & 0.88 & 0.64 & 0.14 \\
\hline RM162 & 0.9 & 0 & 3 & 0.17 & 1.21 & 0.03 & 0.14 & 0.8 & 0.95 & 0.73 & 0.09 \\
\hline RM184 & 0.84 & 4 & 13 & 0.28 & 1.5 & 0.33 & 0.27 & -0.2 & 0.5 & 0.59 & 0.17 \\
\hline RM205 & 0.75 & 2 & 7 & 0.41 & 1.89 & 0.34 & 0.4 & 0.16 & 0.57 & 0.49 & 0.26 \\
\hline RM207 & 0.73 & 7 & 14 & 0.44 & 2.11 & 0.13 & 0.48 & 0.73 & 0.85 & 0.44 & 0.32 \\
\hline RM209 & 0.36 & 5 & 12 & 0.66 & 1.99 & 0.03 & 0.4 & 0.93 & 0.97 & 0.5 & 0.25 \\
\hline RM212 & 0.71 & 2 & 10 & 0.42 & 1.95 & 0.33 & 0.43 & 0.24 & 0.6 & 0.48 & 0.27 \\
\hline RM213 & 0.88 & 2 & 6 & 0.21 & 1.84 & 0.31 & 0.39 & 0.19 & 0.53 & 0.42 & 0.34 \\
\hline RM215 & 0.85 & 2 & 5 & 0.24 & 1.16 & 0.02 & 0.09 & 0.82 & 0.98 & 0.86 & 0.04 \\
\hline RM219 & 0.91 & 0 & 3 & 0.15 & 1.51 & 0 & 0.25 & 1 & 1 & 0.54 & 0.21 \\
\hline $\mathrm{RM} 220$ & 0.47 & 2 & 8 & 0.60 & 1.8 & 0.19 & 0.36 & 0.48 & 0.73 & 0.48 & 0.27 \\
\hline RM224 & 0.4 & 1 & 6 & 0.60 & 1.91 & 0.05 & 0.36 & 0.86 & 0.93 & 0.5 & 0.25 \\
\hline $\mathrm{RM} 225$ & 0.37 & 12 & 19 & 0.72 & 2.08 & 0.31 & 0.47 & 0.35 & 0.63 & 0.42 & 0.34 \\
\hline Mean & 0.66 & 2.18 & 6.49 & 0.41 & 1.54 & 0.12 & 0.27 & 0.62 & 0.83 & 0.59 & 0.2 \\
\hline
\end{tabular}

Where, PIC: Polymorphic Information Content; Ho: Observed Heterozygosity; He: Expected Heterozygosity; $F_{i s}$ : Wrights Fixation Index; $F_{i t}$ : Total Inbreeding Coefficient; $F_{s t}$ : Genetic differentiation.

of genomic DNA, $0.5 \mu \mathrm{l}$ of each of the two primers (at a concentration of $10 \mathrm{mM}$ ), $1.75 \mu \mathrm{l}$ of a $10 \mathrm{X}$ Taq buffer, $0.5 \mu \mathrm{l}$ of a $2.5 \mathrm{mM}$ dNTP mixture, $0.1 \mu \mathrm{l}$ of Taq DNA polymerase and $3 \mu \mathrm{l}$ of double distilled water. The temperature profile used for PCR amplification consisted of a $94{ }^{\circ} \mathrm{C}$ and 5 minutes preheating followed by 34 cycles of denaturation at $94{ }^{\circ} \mathrm{C}$ for $45 \mathrm{~s}$, annealing at $55^{\circ} \mathrm{C}$ to $67^{\circ} \mathrm{C}$ (depending on the annealing temperature of primer) for $30 \mathrm{~s}$, extension at $72{ }^{\circ} \mathrm{C}$ for $30 \mathrm{~s}$, and a final extension at $72{ }^{\circ} \mathrm{C}$ for $7 \mathrm{~min}$.

\section{Polyacrylamide gel electrophoresis, SSR alleles scoring and analysis}

The amplified PCR products $(10 \mu \mathrm{l})$ were mixed with a 3 $\mu \mathrm{l}$ bromophenol blue loading dye and electrophoresed in an 8.0\% polyacrylamide gel and detected using silver staining as described by [31]. The size of the most intensely amplified product was determined based on its migration in comparison with the size standard ( $50 \mathrm{bp}$ DNA ladder). The different alleles of a marker were identified on the basis of their size or length in base pairs (bp) variation. Due to the co-dominant nature of SSR markers, the amplified bands representing the different alleles were scored as different genotypes. Thus, bands were recorded as $(11,22,33$. . ) to represent homozygous genotypes or $(12,13,23 .$.$) to indicate the heterozygous$ genotypes. For each marker, '?' was used for missing data.

The number of alleles per locus, number of rare alleles, expected and observed heterozygosity and Fstatstic values like $F_{\text {is }}, F_{\text {it }}$ and $F_{\text {st }}$ were calculated using GenAlEx 6.502 [33]. Besides, the major allelic frequency and PIC of each marker were computed using Power Marker Version 3.25 [34]. To detect unique alleles, number of accessions having a specific allele was counted for each locus and alleles with a frequency of less than $5 \%$ were considered as unique alleles for a particular locus. According to [35], polymorphic information content (PIC) for each marker was also calculated.

Mathematically,
$\mathrm{Ne}=1 / \sum \mathrm{pi}^{2}$

$\mathrm{Ho}=$ Number of observed heterozygotes/total number of individuals

$$
\begin{aligned}
& H e=1-\sum_{i=1}^{n} p i^{2} \\
& P I C=1-\sum_{i=1}^{n} p i
\end{aligned}
$$

Fis $=($ Mean He - Mean Ho $) /$ Mean He

Fit $=(\mathrm{Ht}-$ Mean $\mathrm{Ho}) / \mathrm{Ht}$

$\mathrm{Fst}=(\mathrm{Ht}-$ Mean $\mathrm{He}) / \mathrm{Ht}$

$\mathrm{Nm}=[(1 /$ Fst $)-1] / 4$

Key; $\mathrm{Pi}$ is the frequency of the $\mathrm{i}^{\text {th }}$ allele, $\sum \mathrm{pi}^{2}$ is the sum of squared allele population frequency, Mean He is average $\mathrm{He}$ across the populations, mean $\mathrm{Ho}$ is average Ho across the populations and $\mathrm{Ht}$ is total expected heterozygosity.

\section{Results}

\section{Overall allelic diversity}

A total of 440 different and reproducible alleles were detected at the 67 microsatellite markers' loci. The number of alleles per locus generated by each marker ranged from 2 to 19 , with an average value of 6.49 . Among those SSR markers, the highest numbers of alleles $(19,14,13,13$ and 12) were produced by RM225, RM207, RM184, RM206 and RM209, respectively and the smallest number of alleles (2) was produced for RM22 and RM171 (Table 5). The frequency of a major allele at each locus ranged from 34\% (RM1) to $95 \%$ (RM60). And on average, $66 \%$ of the total alleles of the 67 SSR markers were common or major alleles.

For each SSR marker, effective number of allels (Ne), observed heterozygocity ( $\mathrm{Ho})$, genetic diversity ( $\mathrm{He})$, Wrights 
fixation index (Fis), Total inbreeding coefficient (Fit), Genetic differentiation (Fst), and gene flow ( $\mathrm{Nm}$ ) were calculated and indicated in (Table 5). The effective number of alleles was 103.84 in total and per locus Ne varied from 0.85 (RM14) to 2.3 (RM1) with an average of 1.54 . When all populations were pooled as one for each locus, the observed hetrozygosity (Ho) was in a range of 0 to 0.5 and 0.12 on average. From the 67 SSR markers-based study, level of genetic diversity (He) fluctuated between 0.06 (RM126) and 0.48 (RM207) with an average of 0.27 .

In this study, the observed hetrozygosity (0.12) was actually less than the expected heterozygosity (0.27). From the entire microsatellite loci assayed in this study, the mean degree of genetic differentiation among populations (Fst), the Wright's fixation index (Fis), Total inbreeding coefficient (Fit), and gene flow ( $\mathrm{Nm}$ ) values were $0.59,0.62,0.83$ and 0.2 , correspondingly (Table 5 ). Here the average fixation index value (0.62) revealed significant deviation from HardyWeinberg expectations or significant heterozygote deficiency.

The degree of genetic differentiation (Fst) range of the whole loci was between 0.21 for RM55 and 0.88 of RM126. This very great mean Fst value (0.59), indicated the high levels of genetic differentiation among the sampled populations. Moreover, indirect estimates of gene flow from this very high Wright's Fst value showed the average number of migrants per generation across the Oryza species $(\mathrm{Nm})$ as 0.2 .

\section{PIC values and unique alleles}

Based on their allelic diversity and frequency, PIC values of the 67 SSR markers showed a very great variation. In this study, the highest PIC value (0.72) was recorded for RM225 and RM1. As shown on Table 5, 0.41 was the average PIC value and RM60 (0.09) and RM 22 (0.1) were rice SSR markers with the lowest PIC values. About $38.9 \%$ of the SSR markers used in this study showed PIC values higher than 0.5 and highly informative.

From the 440 alleles generated by the 67 microsatellite markers, $33.63 \%$ (148 of them) had a frequency $\leq 0.05$ and were detected as rare alleles. The maximum number of unique alleles (12) were found in RM225 and the average of the rare alleles was 2.18 (Table 5). Moreover, $77.8 \%$ of the markers with higher PIC value $(>0.60)$ had at least one unique alleles. Generally, RM207 and RM225 were the most informative markers as, they identified rare alleles and produced the highest number of alleles (14 and 19), respectively.

\section{Discussion}

Microsatellites are PCR based DNA markers that are widely used in genetic diversity, varietal identification, and germplasm characterization of rice [36]. However, their distribution across the genome and level of polymorphism is quite different among each other [37]. Hence, their application on rice germplasm characterization and improvement will depend on reliability of the information they provide [6]. This comparative evaluation study of the rice SSR markers polymorphism also showed differences on their power of revealing genetic variation over diverse set of Oryza species and subspecies.

Differences in plant material composition, population size and species composition make direct comparisons of this study with others a bit irrational. This study however, estimates genetic diversity of each marker in terms of parameters like; the mean number of alleles, genetic diversity, PIC values and number of rare alleles over different Oryza species.

The mean number of alleles detected in the present study (6.49) is comparable to the number of alleles noted by earlier researchers on African rice [38]. According to [39], number of alleles in African wild rice (from 11 to 16 with an average of 14 alleles) is high. Thus, inclusion of large African wild rice materials in this study could lead to greater diversity than the results observed in previous studies showing 1-8 alleles with an average of 4.58 alleles for various classes of microsatellite [40] and also 3 to 9 alleles, with an average of 4.53 alleles per locus for 30 microsatellite markers [41]. Other than the diverse Oryza species assessed in this study, such variability on the number of detected alleles per locus might be associated to the markers' specificity [1].

In this study, all of the markers with highest number of allels $(\mathrm{RM} 225=19, \mathrm{RM} 207=14, \mathrm{RM} 184=13, \mathrm{RM} 206=13$ and RM209 = 12) have a dinucleotide repeat motifs of $(C T) 18$, (CT)25, (GA)19, (CA)7 and (CT)18, respectively. Though 3 of those 5 markers were with (CT) motifs, [27] reported markers with repeat motif (GA) displayed high level of variation among the rice genotypes than markers with (CT) motifs. However, our results showed that markers having perfect dinucleotide repeat motifs irrespective of their dinucleotide type such as; $(C T),(G A)$ or (CA)), are potentially best markers for molecular characterization and diversity analysis of different Oryza species.

The effective number of alleles in this study was 103.84 in total and per locus varied from 0.85 (RM14) to 2.3 (RM1) with an average of 1.54 . Here the range was not wide as, the actual number of alleles varying between 2 and 19. This fact implicated the high influence of number of tested samples on the number of identified alleles and its insignificant impact on the effective number of alleles. It also reveals the higher reliability of effective number of alleles for practical genetic diversity analysis [42-44]. From the whole SSR markers assessed in this study, RM225, RM209, RM207 and RM84 produced Na greater than 10 and Ne greater than 2. Since such markers showed greater genetic diversity, they could be suitable tools for assessing genetic diversity within and among Oryza members. The highest levels of actual and effective number of alleles recorded in such locus also contribute to their great levels of expected heterozygosity (on average 0.46) [45].

However, all loci in this study except RM159 and RM184 showed a lower observed Heterozygosity $(\mathrm{Ho})$ (mean = 0.12 ) than the expected Heterozygosity $(\mathrm{He})$ (mean $=0.27$ ), suggesting a clear shift from the Hardy-Weinberg equilibrium [46] and this shift can be attributed to forces akin to inbreeding within groups [47] or lack of distinctly isolated Oryza populations [46]. Such heterozygous deficiency or deviations from HardyWeinberg expectation were also indicated by the relatively high $F_{\text {is }}$ value (0.62). The average $F_{\text {st }}=0.59$, implicated $59 \%$ of the total genetic variation among populations. The mating 
system and its consequence of high intrapopulation inbreeding $\left(F_{i s}=0.62\right)$ could be major factors for the high total inbreeding $\left(F_{i t}=0.83\right)$ [48]. In the present study, low value for the number of migrants per generation ( $\mathrm{Nm}=0.2)$ was estimated. In fact, pollen viability in the genus Oryza is in general limited to few minutes [49]. Thus, dispersal of whole plants by programmed abscission followed by floating downstream might be factors responsible for the observed $\mathrm{Nm}$ value.

According to [50], PIC value is a derivative of both allelic diversity and frequency. Sensitivity of genotyping method and location of primers in the genome largely affect PIC [6]. Thus, PIC value that reflects the allele frequency and diversity among accessions could be varied from one to another SSR locus [51]. In the present study, PIC values for the 67 SSR markers ranged from the lowest value of 0.09 (RM60) to the highest value of 0.72 (RM1 and RM225), with a mean of 0.41 (Table 5). This level of mean polymorphism (0.41) is consistent with the reported PIC value in previous works [2,52,53].

According to [54], markers with a PIC value more than 0.5 are regarded as highly polymorphic. About $38.9 \%$ of the SSR markers used in this study had PIC values exceeding 0.5 (Table 5). Such great PIC values maybe associated to the highly co-dominant expression or presence of multiple alleles [19]. The highly polymorphic markers implicated in this study are greatly informative for genetic studies and detection of more alleles at a specific locus [55].

These days, number of unique alleles in a population (private allelic richness) is largely considered for many conservation and population genetics applications [56], distinguishing different species and populations of a species [57] and inferring evolutionary history of a population [58]. As indicated in Table 5, the maximum and average numbers of unique alleles in this study were 12 (RM225) and 2.18 respectively. This wide variation in the numbers of private alleles might show the variable periods of genetic isolation during the evolutionary history of Oryza species [48]. Markers used in this study and their association with rare alleles could be utilized by plant breeders and geneticists for the marker assisted selection programs [5]. Moreover, possible relationship of such unique alleles with diverse quantitative trait locus (QTL) regions must be studied [51].

\section{Acknowledgement}

This work was supported by grants from the National Key R\&D Program of China (2017YFD0100201), Yunnan National Foundation of Science.

\section{References}

1. Sajib MA, Hossain M, Mosnaz JA, et al. (2012) SSR marker-based molecular characterization and genetic diversity analysis of aromatic landreces of rice (Oryza sativa L.). J BioSci Biotech 1: 107-116.

2. Lu H, Redus MA, Coburn JR, et al. (2005) Population structure and breeding patterns of 145 U.S. rice cultivars based on SSR marker analysis. Crop Sci 45: 66-76.

3. Garris AJ, Tai TH, Coburn J, et al. (2005) Genetic structure and diversity in Oryza sativa L. Genetics 169: 1631-1638.

4. Khush GS (1997) Origin, dispersal, cultivation, and variation of rice. Plant Mol Biol 35: 25-34.

5. Aliyu R, Adamu AK, Gregorio GB (2013) Allelic data revealing interrelatedness in rice species (Oryza sativa, Oryza glaberrima, Oryza barthii) and the interspecific hybrids (NERICA). Journal of Plant Breeding and Crop Science 5: 12-19.

6. Singh N, Choudhury DR, Singh AK, et al. (2013) Comparison of SSR and SNP markers in estimation of genetic diversity and population structure of Indian rice varieties. PLoS ONE 8: e84136.

7. Virk PS, Newbury JH, Bryan GJ, et al. (2000) Are mapped or anonymous markers more useful for assessing genetic diversity? Theor Appl Genet 100: 607-613.

8. Song ZP, Xu X, Wang B, et al. (2003) Genetic diversity in the northernmost Oryza rufipogon populations estimated by SSR markers. Theor Appl Genet 107: 1492-1499.

9. Teixeira da Silva JA (2005) Molecular markers for phylogeny, breeding and ecology in agriculture. In: Thangadurai D, Pullaiah T, Tripathy L, Genetic resources and biotechnology vol III. Regency Publications, New Delhi, India, 221-256.

10. Olufowote J, Xu YX, Chen X, et al. (1997) Comparative evolution of within-cultivar variation of rice (Oryza sativa L.) using microsatellites and RFLPs markers. Genome 40: 370-378.

11. Akagi HY, Yokozola A, Fujimura T (1997) Highly polymorphic microsatellites of rice consist of AT repeats, and a classification of closely related cultivars with these microsatellite loci. Theor Appl Genet 94: 61-67.

12. Schoen DJ, Brown AD (1993) Maximising allelic diversity in core collection of wild crop relatives. The role of genetic markers. In: Hodgkinetal T, Core collections of plant genetic resources. John wiley and son, Chichester, UK, 55-76.

13. O'Hanlon PC, Peakall R, Briese DT (2000) A review of new PCRbased genetic markers and their utility to weed ecology. Weed Research 40: 239-254.

14. Rahman ML, Jiang W, Chu SH, et al. (2009) High resolution mapping of two rice brown plant hopper resistance genes, $\mathrm{Bph} 20(\mathrm{t})$ and $\mathrm{Bph} 21(\mathrm{t})$, originating from Oryza minuta. Theor Appl Genet 119: 1237-1246.

15. Kalia RK, Rai MK, Kalia S, et al. (2011) Microsatellite markers: an overview of the recent progress in plants. Euphytica 177: 309-334.

16. Provan J, Powell W, Hollingsworth PM (2001) Chloroplast microsatellites: New tools for studies in plant ecology and evolution. Trends Ecol Evol 16: 142-147.

17. Chung AM, Staub JE, Chen JF (2006) Molecular phylogeny of Cucumis species as revealed by consensus chloroplast SSR marker length and sequence variation. Genome 49: 219-229.

18. Rajendrakumar P, Biswal AK, Balachandran SM, et al. (2007) Simple sequence repeats in organellar genomes of rice: Frequency and distribution in genic and intergenic regions. Bioinformatics 23: 1-4.

19. Etemad A, Maziah M, Daud KS (2012) Determination of genetic relatedness among selected rice (Oryza sativa, L.) cultivars using microsatellite markers. Afri J Biotech 11: 7158-7165.

20. IRGSP (2005) The map-based sequence of the rice genome. Nature 436: 793-800.

21. Zhu XC, Wu HW, Raman H, et al. (2012) Evaluation of simple sequence repeat (SSR) markers from Solanum crop species for Solanum elaeagnifolium. Weed Research 52: 217-223.

22. Zhang SB, Zhu Z, Zhao L, et al. (2007) Identification of SSR markers closely linked to eui gene in rice. Yi Chuan 29: 365-370.

23. Ma H, Yin Y, Guo ZF, et al. (2011) Establishment of DNA fingerprinting of Liaojing series of japonica rice. Middle-East J Sci res 8: 384-392. 
24. Parida SK, Kalia SK, Sunita K, et al. (2009) Informative genomic microsatellite markers for efficient genotyping applications in sugarcane. Theor Appl Genet 118: 327-338.

25. Xiao XY, Wang YP, Zhang JY, et al. (2006) SSR marker-based genetic diversity fingerprinting of hybrid rice in Sichuan, China. Chin J Rice Sci 20: 1-7.

26. Peng ST, Zhuang JY, Yan QC, et al. (2003) SSR markers selection and purity detection of major hybrid rice combinations and their parents in China. Zhongguo Shuidao Kexue 17: 1-5.

27. Temnykh S, Park WD, Ayers N, et al. (2000) Mapping and genome organization of microsatellite sequences in rice (Oryza sativa $\mathrm{L}$ ). Theor Appl Genet 100: 697-712.

28. McCouch SR, Temnykh S, Lukashova A, et al. (2001) Rice Genetics IV. In: Microsatellite markers in rice abundance, diversity and applications. IRRI, Manila, Philippines, 117-135.

29. Rabbani MA, Pervaiz ZH, Masood MS (2008) Genetic diversity analysis of traditional and improved cultivars of Pakistani rice (Oryza sativa L.) using RAPD markers. Elec J Biotech 11: 1-10.

30. Doyle JJ, Doyle JL (1987) A rapid DNA isolation procedure for small quantities of fresh leaf tissue. Phytochem Bull 19: 11-15.

31. Panaud O, Chen X, McCouch SR (1996) Development of microsatellite. markers and characterization of simple sequence length polymorphism (SSLP) in rice (Oryza sativa L.). Mol Gen Genet 252: 597-607.

32. Chen X, Temnykh S, Xu Y, et al. (1997) Development of a microsatellite framework map providing genome-wide coverage in rice (Oryza sativa L). Theor Appl Genet 95: 553-567.

33. Peakall R, Smouse PE (2012) GenAlEx 6.5: genetic analysis in excel. Population genetic software for teaching and research-an update. Bioinformatics 28: 2537-2539.

34. Liu K, Muse SV (2005) Power marker: An integrated analysis environment for genetic marker analysis. Bioinformatics 21: 2128-2129.

35. Peng JH, Lapitan NL (2005) Characterization of EST-derived microsatellites in the wheat genome and development of eSSR markers. Funct Inter Genomics 5: 8096.

36. Yu GQ, Bao Y, Shi $\mathrm{CH}$, et al. (2005) Genetic diversity and population differentiation of Liaoning weedy rice detected by RAPD and SSR markers. Biochem Genet 43: 261-270.

37. Cho GY, Ishii T, Temnykh S, et al. (2000) Diversity of microsatellites derived from genomic libraries and GenBank sequences in rice (Oryza sativa L). Theor Appl Genet 100: 713-722.

38. Drame KE, Sanchez I, Gregorio G, et al. (2011) Suitability of a selected set of simple sequence repeats (SSR) markers for multiplexing and rapid molecular characterization of African rice (Oryza glaberrima Steud.). Afr J Biotech 10: 6675-6685.

39. Getachew M, Teklehaimanot H, Tileye F, et al. (2013) Genetic diversity of wild rice Oryza longistaminata Chev. Et. Roher in Ethiopia as revealed by microsatellite markers. Genet Resour 60: 1047-1056.

40. Siwach P, Jain S, Saini N, et al. (2004) Allelic diversity among Basmati and non-Basmati long-grain indica rice varieties using microsatellite markers. Journal of Plant Biochemistry and Biotechnology 13: 25-32.

41. Hossain MZ, Rasul MG, Ali MS, et al. (2007) Molecular characterization and genetic diversity in fine grain and aromatic landraces of rice using microsatellite markers. Bangladesh J
Genet PI Breed 20: 1-10.

42. Liu J, Guan JP, Xu DX, et al. (2008) Analysis of genetic diversity and population structure in lentil (Lens culinaris Medik) germplasm by SSR markers. Acta Agron Sin 34: 1901-1909.

43. Zong XX, Guan JP, Wang SM, et al. (2008) Genetic diversity among Chinese pea (Pisum sativum L.) landraces revealed by SSR markers. Acta Agron Sin 34: 1330-1338.

44. Zong XX, Rebecca F, Robert RR, et al. (2009) Identification and analysis of genetic diversity structure within Pisum genus based on microsatellite markers. Sci Agric Sin 42: 36-46.

45. Al-Faifi AS, Migdadi MH, Algamdi SS, et al. (2016) Development, characterization and use of genomic SSR markers for assessment of genetic diversity in some Saudi date palm (Phoenix dactylifera L.) cultivars. Elect J Biotech 21: 18-25.

46. Oloka MB, Lamol J, Rubaihayo P, et al. (2015) The use of multiplexed simple sequence repeat (SSR) markers for analysis of genetic diversity in African rice genotypes. Afri J Biotech 14: 1533-1542.

47. Masudaab M, Throwerab F, Nicholas KM (2009) The effects of violating hardy-weinberg equillibrium assumptions on a clusterbased population mixture analysis of stealhead populations in Southeast Alaska. N Am J Fish Manage 29: 140-150.

48. Karasawa MG, Vencovsky R, Silva MC, et al. (2007) Genetic structure of Brazilian wild rice (Oryza glumaepatula Steud., Poaceae) populations analyzed using microsatellite markers. Genet Mol Biol 30: 400-410.

49. Oka HI (1988) Origin of Cultivated Rice. Japan Scientific Societies Press, Tokyo, and Elsevier Science Publishers, Amsterdam, Netherlands, 254.

50. Pervaiz ZH, Rabbani MA, Pearce SR, et al. (2009) Determination of genetic variability of Asian rice (Oryza sativa L.) varieties using microsatellite markers. Afr J Biotech 8: 5641-5651.

51. Vu TH, Nguyen TH, Tran DK, et al. (2016) Genetic diversity of Vietnamese lowland rice germplasms as revealed by SSR markers in relation to seedling vigour under submergence. Biotech \& Biotech Equip 30: 17-25.

52. Wong SC, Yiu PH, Bong SW, et al. (2009) Analysis of Sarawak Bario rice diversity using microsatellite markers. Am J Agri Biol Sci 4: 298-304.

53. Hossain MM, Islam MM, Hossain H, et al. (2012) Genetic diversity analysis of aromatic landraces of rice (Oryza sativa L.) by microsatellite markers. Genes, Genomes and Genomics 6: 42-47.

54. Botstein D, White RL, Skolnick M, et al. (1980) Construction of genetic linkage map in man using restriction fragment length polymorphisms. Am J Hum Genet 32: 314-331.

55. Dewoody JA, Honeycutt RL, Skow LC (1995) Microsatellite markers in white-tailed deer. J Hered 86: 317-319.

56. Kalinowski ST (2004) Counting alleles with rarefaction: private alleles and hierarchical sampling designs. Conserv Genet 5: 539-543.

57. Bashyal A, Gros BA, Venegas-Anaya M, et al. (2014) Assessment of microsatellites in estimating inter-and intraspecific variation among Neotropical Crocodylus species. Genet Mol Res 13: 5492-5502.

58. Castric V, Bernatchez L (2003) The rise and fall of isolation by distance in the anadromous brook charr (Salvelinus fontinalis Mitchill). Genetics 163: 983-996.

DOI: $10.36959 / 973 / 418$

Copyright: (C) 2018 Melaku G, et al. This is an open-access article distributed under the terms of the Creative Commons Attribution License, which permits unrestricted use, distribution, and reproduction in any medium, provided the original author and source are credited. 